\title{
Orthogonality and characterizations of inner product spaces
}

\section{O.P. Kapoor and Jagadish Prasad}

\begin{abstract}
Using the notions of orthogonality in normed linear spaces such as isosceles, pythagorean, and Birkhoff-James orthogonality, in this paper we provide some new characterizations of inner product spaces besides giving simpler proofs of existing similar characterizations. In addition we prove that in a normed linear space pythagorean orthogonality is unique and that isosceles orthogonality is unique if and only if the space is strictly convex.
\end{abstract}

\section{Introduction}

A normed linear space is called an inner product space if there is an inner product $(x, y)$ defined in it such that $\|x\|^{2}=(x, x)$. There is a long list of conditions on the norm, called norm postulates, strong enough to characterize inner product spaces amongst normed linear spaces. The best known norm postulate is by Jordan and von Neumann [7],

$$
\text { (JN) } x, y \in X,\|x+y\|^{2}+\|x-y\|^{2}=2\left(\|x\|^{2}+\|y\|^{2}\right) .
$$

The other norm postulates needed here are

$$
\begin{aligned}
& \text { (D) if } x, y \in x,\|x\|=\|y\|=1 \text {, then }\|x+y\|^{2}+\|x-y\|^{2}=4[1] \text {; } \\
& (D, \sim) \text { if }\|x\|=\|y\|=1 \text {, then there exist } \alpha \text { and } B \text { with } \\
& 0<\alpha<1,0<\beta<1 \text {, such that }
\end{aligned}
$$

Received 4 October 1978. 
$\beta(1-\beta)\|\alpha x+(1-\alpha) y\|^{2}+\alpha(1-\alpha)\|\beta x-(1-\beta) y\|^{2} \sim[\alpha+\beta-2 \alpha \beta][\alpha \beta+(1-\alpha)(1-\beta)]$

[2];

(J) if $\|x+y\|=\|x-y\|$, then $\|x+k y\|=\|x-k y\|$ for every real $k$ [5];

(J') if $\|x+y\|^{2}=\|x\|^{2}+\|y\|^{2}$ then $\|x+k y\|^{2}=\|x\|^{2}+\|k y\|^{2}$ for every $k$ [5];

$\left(L_{1}\right)$ there is a fixed real $\alpha \neq 0, \pm 1$ such that $x, y \in X$ and $\|x+y\|=\|x-y\|$ imply $\|x+\alpha y\|=\|x-\alpha y\| \quad[8]$.

The main purpose of this paper is to give some new characterizations of inner product spaces and to provide simpler proofs of existing similar characterizations. Before proceeding further we give below basic definitions and notations for convenience of reference. Throughout this paper, $X$ is a real normed linear space. $X$ is called strictly convex if $0<k<1, x \neq y$ imply $\|k x+(1-k) y\|<k\|x\|+(1-k)\|y\|$. If $x, y \in X$, then $x$ is called isosceles orthogonal to $y \quad\left(x L_{i} y\right)$ if $\|x+y\|=\|x-y\|$, $x$ is called pythagorean orthogonal to $y \quad\left(x \perp_{p} y\right)$ if $\|x+y\|^{2}=\|x\|^{2}+\|y\|^{2}$ and $x$ is Birkhoff-James orthogonal to $y\left(x \perp_{J} y\right)$ if $\|x+k y\| \geq\|x\|$ for all real $k$. For details of these orthogonalities one can refer to James [5], [6]. The following results about these orthogonalities will be useful for us.

THEOREM 1 (James [5], [6]). If $x \neq 0, y \in X$, then there exist numbers $a, b, c$, and $d$, such that $x \perp_{\mathrm{i}} a x+y, x \perp_{\mathrm{p}} b x+y$, $x \perp_{J} c x+y$, and $d x+y \perp_{J} x$. Further, if $\|y\| \leq\|x\|$, then $|a| \leq\|y\| /\|x\|$.

An orthogonality $\perp$ is called left (right) unique if for $x \neq 0$, $y \in X$, there exist only one $a$ such that $x \perp a x+y(a x+y \perp x)$. For isosceles and pythagorean orthogonalities, right and left uniqueness are equivalent. For Birkhoff-James orthogonality, the following was proved.

THEOREM 2 (James [6]). Birkhoff-James orthogonality is left (right) unique if and only if $X$ is strictly convex (smooth). 


\section{Uniqueness of isosceles and pythagorean orthogonality}

THEOREM 3. (i) Isosceles orthogonality in $X$ is unique if and only if $X$ is strictly convex.

(ii) Pythagorean orthogonality is unique.

Proof. (i) Suppose $X$ is strictly convex and isosceles orthogonality is not unique. It can be easily seen that there nust exist $x \neq 0, y \in X$, and a number $a>0$, such that $x \perp_{i} y$ and $x \perp_{i} \alpha x+y$. The function $q(t)=\|y+t x\|,-\infty<t<\infty$, is a strictly convex function with $q(1)=q(-1)$ and $q(\alpha+1)=q(\alpha-1)$. In the case $0<\alpha \leq 2$ we see that

$$
\begin{aligned}
q(\alpha-1) & =q\left(\frac{2-\alpha}{2}(-1)+\frac{\alpha}{2}\right)<q(1) \\
& =q\left(\frac{\alpha}{2}(\alpha-1)+\left(1-\frac{\alpha}{2}\right)(\alpha+1)\right)<q(\alpha+1),
\end{aligned}
$$

and that yields a contradiction. In the case $\alpha>2, q$ will have two distinct local minima - one each in the intervals $[-1,1]$ and $[\alpha-1, \alpha+1]$. But $q$ is strictly convex and thus can have only one point of minimum - a global minimum. Again a contradiction is seen. To prove the other way we start with the assumption that $X$ is not strictly convex. So let $x \neq y \in X$ such that $\|x\|=\|y\|=\|x+y\| / 2=1$. Then

$$
\|x+y\|=\|x+y+x-y\|=\|(x+y)-(x-y)\|,
$$

or

$$
\left\|x^{\prime}\right\|=\left\|x^{\prime}+y^{\prime}\right\|=\left\|x^{\prime}-y^{\prime}\right\|,
$$

where

$$
x^{\prime}=x+y \text { and } y^{\prime}=x-y \neq 0 \text {. }
$$

Then we have

$$
\left\|x^{\prime}+\left(y^{\prime} / 2\right)-\left(y^{\prime} / 2\right)\right\|=\left\|x^{\prime}+\left(y^{\prime} / 2\right)+\left(y^{\prime} / 2\right)\right\|=\left\|x^{\prime}-\left(y^{\prime} / 2\right)-\left(y^{\prime} / 2\right)\right\| .
$$

Thus

$$
y^{\prime / 2} \perp_{i} x^{\prime}+y^{\prime / 2} \text {, and } y^{\prime / 2} \perp_{i} x^{\prime}-y^{\prime / 2} \text {, }
$$

and therefore the isosceles orthogonality is not unique. That completes the proof of part (i). 
(ii) Let us assume that pythagorean orthogonality is not unique. Then we must have elements $x \neq 0$ and $y \in X$, and a number $\alpha>0$ such that $x \perp_{\mathrm{p}} y$ and $x \perp_{\mathrm{p}} \alpha x+y$; that is

$$
\|x+y\|^{2}=\|x\|^{2}+\|y\|^{2} \text { and }\|x+\alpha x+y\|^{2}=\|x\|^{2}+\|\alpha x+y\|^{2} .
$$

Setting $g(t)=\|y+t x\|^{2}$ we have, by (1),

$$
\begin{gathered}
g(1)=\|x\|^{2}+g(0), \\
g(\alpha+1)=\|x\|^{2}+g(\alpha) .
\end{gathered}
$$

Clearly $g$ is a convex function on $-\infty<t<\infty$. Let us first prove that for $0<t<1$ and $s_{1}$ and $s_{2}$ such that $g\left(s_{1}\right) \neq g\left(s_{2}\right)$ we have

$$
g\left(t s_{1}+(1-t) s_{2}\right)<t g\left(s_{1}\right)+(1-t) g\left(s_{2}\right) \text {. }
$$

$g\left(t s_{1}+(1-t) s_{2}\right)=\left\|t\left(y+s_{1} x\right)+(1-t)\left(y+s_{2} x\right)\right\|^{2}$

$$
\begin{aligned}
& \leq t^{2}\left\|y+s_{1} x\right\|^{2}+(1-t)^{2}\left\|y+s_{2} x\right\|^{2}+2 t(1-t)\left\|y+s_{1} x\right\|\left\|y+s_{2} x\right\| \\
& =t\left\|y+s_{1} x\right\|^{2}+(1-t)\left\|y+s_{2} x\right\|^{2}+\left(t^{2}-t\right) \\
& \times\left[\left\|y+s_{1} x\right\|^{2}+\left\|y+s_{2} x\right\|^{2}-2\left\|y+s_{1} x\right\|\left\|y+s_{2} x\right\|\right] \\
& =\operatorname{tg}\left(s_{1}\right)+(1-t) g\left(s_{2}\right)-t(1-t)\|\| y+s_{1} x\|-\| y+s_{2} x \|\left.\right|^{2} \\
& \leq \operatorname{tg}\left(s_{1}\right)+(1-t) g\left(s_{2}\right) \text {, }
\end{aligned}
$$

where the inequalities will be strict if $g\left(s_{1}\right) \neq g\left(s_{2}\right)$. That proves (4).

Now suppose $0<\alpha<1$; we get by using (2), (3), and (4),

$$
\begin{aligned}
g(\alpha) & <\alpha g(1)+(1-\alpha) g(0), \\
g(1) & <\alpha g(\alpha)+(1-\alpha) g(\alpha+1) \\
& =\alpha g(\alpha)+(1-\alpha)(g(\alpha)+g(1)-g(0)),
\end{aligned}
$$

and that yields

$$
\alpha g(1)+(1-\alpha) g(0)<g(\alpha),
$$

contradicting (5).

In the case $\alpha>1$, we use convexity of $g$, and (2) and (3) to 
obtain $g(0) \neq g(\alpha)$ and $g(1) \neq g(\alpha+1)$, and then by (4) we get

$$
g(1)<\frac{\alpha-1}{\alpha} g(0)+\frac{1}{\alpha} g(\alpha)
$$

and

$$
\begin{aligned}
g(\alpha) & <\frac{g(1)}{\alpha}+\frac{\alpha-1}{\alpha} g(\alpha+1) \\
& =\frac{g(1)}{\alpha}+\frac{\alpha-1}{\alpha}(g(\alpha)+g(1)-g(0)),
\end{aligned}
$$

contradicting (7). In the case $\alpha=1$ we have

$$
g(2)=g(1)+\|x\|^{2}=g(0)+2\|x\|^{2}
$$

and

$$
g(1)<\frac{1}{2}(g(0)+g(2))=g(0)+\|x\|^{2},
$$

which is false. Thus in all cases we get a contradiction. Hence pythagorean orthogonality is unique in any normed linear space.

\section{Characterizations of inner product spaces}

We begin with a theorem that combines two results of Day ([1], Theorems 5.1, 5.2), characterizing inner product spaces.

THEOREM 4. For a normed linear space $X$ the following are equivalent:

(i) $X$ is an irner product space;

$$
\text { (ii) } x, y \in X, x \perp_{\mathrm{p}} y \Rightarrow x \perp_{\mathrm{i}} y \text {; }
$$

(iii) $x, y \in X, x \perp_{i} y \Rightarrow x \perp_{p} y$.

Proof. $(i) \Rightarrow(i i)$ is obvious. To show $(i i) \Rightarrow$ (iii) let us first prove that if ( $i i$ ) holds then $X$ is strictly convex. If not then there exist $x \neq y \in X$ such that $\|x\|=\|y\|=\frac{\|x-y\|}{2}=1, x 1_{p} y$. By Theorem $I$ there exists a number $\alpha \neq 0$ such that $x 1_{p} \alpha x+y$; that is

$$
\|x+\alpha x+y\|^{2}=\|\alpha x+y\|^{2}+\|x\|^{2}=1+\|\alpha x+y\|^{2} .
$$

Therefore 


$$
\|(1+\alpha) x+y\|=\|(1-\alpha) x+y\| .
$$

By Theorem 1, $|\alpha| \leq 1$. From equation (9) we get

$$
(2+\alpha)^{2}=(2+\alpha)^{2}\left\|\frac{(1+\alpha) x+y}{2+\alpha}\right\|^{2}=1+\|\alpha x+y\|^{2} \geq 1 .
$$

That means $\alpha \geq-1$. Thus $\alpha=-1$. Then, from (9),

$$
1=\|y\|^{2}=\|x-y\|^{2}+1,
$$

which contradicts the assumption that $x \neq y$. Thus $X$ is strictly convex. Now suppose that $(i i)$ does not imply (iii). There exist points $x$ and $y$ such that $x \perp_{i} y$ but $x 1_{p} y$. Choose $\alpha \neq 0$ such that $x \perp_{p} \alpha x+y$. But then, by (ii), $x \perp_{i} \alpha x+y$. Thus $x \perp_{i} y$ and $x \perp_{i} \alpha x+y$, contradicting the uniqueness of isosceles orthogonality in strictly convex spaces proved in Theorem 3 above.

To prove (iii) implies (i), we employ the reasoning of ([1], Theorem 5.1). Let $\|x\|=\|y\|=1$. Then $x+y L_{i} x-y$ and, therefore,

$$
\|x+y\|^{2}+\|x-y\|^{2}=\|x+y+x-y\|^{2}=4 .
$$

The result follows from $\left(D_{1}\right)$.

THEOREM 5. For a normed linear space $X$ the following are equivalent:

(i) $X$ is an inner product space;

$$
\text { (ii) } x, y \in X, \quad x \perp_{\mathrm{p}} y \Rightarrow x \perp_{\mathrm{J}} y \text {; }
$$$$
\text { (iii) } x, y \in X, x \perp_{J} y \Rightarrow x \perp_{p} y \text {. }
$$

Proof. (i) $\Rightarrow$ (ii) is straight. To prove $(i i) \Rightarrow$ (iii) we first show that if ( $i i)$ holds then $X$ is strictly convex. If not, let $x \neq y$ such that $\|x\|=\|y\|=\|(x+y) / 2\|=1, x 1_{p}(x+y) / 2$. There exists $\alpha \neq 0$ such that $\frac{x+y}{2} \perp_{p} \alpha \frac{x+y}{2}+x$. Then

$$
\left\|(\alpha+1) \frac{x+y}{2}+x\right\|^{2}=1+\left\|\alpha \frac{(x+y)}{2}+x\right\|^{2}
$$

and, therefore, 


$$
\left\|\frac{x+y}{2}+\alpha k\left(\frac{x+y}{2}\right)+k x\right\| \geq\left\|\frac{x+y}{2}\right\|=1 \text { for all real } k \text {. }
$$

With $k=-1 / \alpha$, equation (12) yields $|\alpha| \leq 1$. Again putting $k=-\frac{1}{\alpha+2}$ in equation (12) and using the fact that every convex combination of $x$ and $y$ is of norm 1 , gives $\left|\frac{1}{\alpha+2}\right| \geq 1$. Thus $\alpha=-1$, and therefore

$$
1=\left\|\frac{x+y}{2}+\frac{x-y}{2}\right\|^{2}=\left\|\frac{x+y}{2}\right\|^{2}+\left\|\frac{x-y}{2}\right\|^{2}=1+\left\|\frac{x-y}{2}\right\|^{2} .
$$

Hence $x=y$ which is a contradiction. $X$ must therefore be strictly convex. Suppose that (ii) does not imply ( $i i i)$. Let $x$ and $y \in X$ such that $x \perp_{J} y$ but $x \underline{d}_{p} y$. But then there exists a number $\alpha \neq 0$ such that $\alpha y+x \perp_{p} y$. By (ii), $\alpha y+x \perp_{J} y$, but that contradicts the left uniqueness of Birkhoff-James orthogonality in strictly convex spaces stated in Theorem 2 above. Hence $(i i) \Rightarrow(i i i)$.

We now prove $(i i i) \Rightarrow(i)$. Let $\|x\|=\|y\|=1$. If $x \perp_{\mathrm{J}} y$ and $x+y \perp_{\mathrm{J}} x-y$, then

$$
4=\|x+y+x-y\|^{2}=\|x+y\|^{2}+\|x-y\|^{2} .
$$

If $x 1_{\mathrm{J}} y$, then choose $z \in X$ such that $x 1_{\mathrm{J}} z$ and $x+z \perp_{\mathrm{J}} x-z$. This choice is possible (see Sundaresan ([9], Lemma 1)). Then

$$
\begin{aligned}
\|z\|^{2}=\left\|\frac{(x+z)-(x-z)}{2}\right\|^{2} & =\left\|\frac{x+z}{2}\right\|^{2}+\left\|\frac{x-z}{2}\right\|^{2} \\
& =\left\|\frac{x}{2}\right\|^{2}+\left\|\frac{z}{2}\right\|^{2}+\left\|\frac{x}{2}\right\|^{2}+\left\|\frac{z}{2}\right\|^{2} .
\end{aligned}
$$

That means $\|x\|=\|z\|=1$. Let $\alpha$ and $\beta$ be such that $y=\alpha x+\beta z$. Then

$$
\begin{aligned}
\|y\|^{2} & =\|\alpha x+\beta z\|^{2}=\alpha^{2}+\beta^{2}, \\
\|x+y\|^{2} & =\|(1+\alpha) x+\beta z\|^{2}=(1+\alpha)^{2}+\beta^{2}, \\
\|x-y\|^{2} & =\|(1-\alpha) x-\beta z\|^{2}=(1-\alpha)^{2}+\beta^{2} .
\end{aligned}
$$

Thus again we see that

$$
\|x+y\|^{2}+\|x-y\|^{2}=2\left(\alpha^{2}+\beta^{2}\right)+2=4 .
$$


Day's criterion $\left(D_{1}\right)$ gives the result.

Lorch [8] proved the criterion $\left(L_{1}\right)$ mentioned in the beginning, which was a considerably weakened form of the criterion $(J)$. We give below a similarly weakened form of ( $\left.\mathrm{J}^{\prime}\right)$.

COROLLARY 6. A normed linear space $X$ is an inner product space if and only if

( $\left.L_{i}^{\prime}\right)$ for some fixed $\alpha \neq 0,1$,

$$
\|x+y\|^{2}=\|x\|^{2}+\|y\|^{2} \Rightarrow\|x+\alpha y\|^{2}=\|x\|^{2}+\|\alpha y\|^{2} .
$$

Proof. The case of $\alpha=-1$ has been proved by Day ([1], Lemma 5.3). Without Ioss of generality we can assume that $\alpha>1$. Suppose $x$ and $y \in X$ are such that $\|x+y\|^{2}=\|x\|^{2}+\|y\|^{2}$. By repeated application of (Li) we will have, for all $n \geq 1$,

$$
\left\|y+\alpha^{n} x\right\|^{2}=\|y\|^{2}+\left\|\alpha^{n} x\right\|^{2} .
$$

Then, for all $n \geq 1$,

$$
\left(\left\|x+\frac{1}{\alpha^{n}} y\right\|^{2}-\|x\|^{2}\right) /\left(1 / \alpha^{n}\right)=\frac{\|y\|^{2}}{\alpha^{n}} .
$$

In the limit we obtain, from (13),

$$
2\|x\| N_{+}(x, y)=0,
$$

where $N_{+}(x, y)$ is the right-hand Gâteaux derivative of the norm functional at $x$ in the direction of $y$. Equation (14) shows that $x \perp_{J} y$ (see James [6]). Thus $x \perp_{\mathrm{p}} y \Rightarrow x \perp_{\mathrm{J}} y$. Therefore $x$ is an inner product space by Theorem 5. The necessity of $\left(L_{1}^{\prime}\right)$ is easy.

To complete the picture we have the following:

THEOREM 7. For a normed linear space $X$ the following are equivalent:

(i) $X$ is an inner product space;

(ii) $x, y \in X, x \perp_{J} y \Rightarrow x \perp_{i} y$;

(iii) $x, y \in X, x \perp_{i} y \Rightarrow x \perp_{J} y$.

Proof. (i) $\Rightarrow$ (ii) and ( $i i i)$ is straight. Suppose (ii) holds. Let 
$x \neq 0, y \in X$. Choose $\alpha$ such that $x \perp_{J} \alpha x+y$. But then $x \perp_{J} k(\alpha x+y)$ for all $k$, because Birkhoff-James orthogonality is homogeneous. By $(i i), x \perp_{i} k(\alpha x+y)$ for all $k$. Thus, by Corollary 4.7 of [5], we have $(i i) \Rightarrow(i)$. To prove the $(i i i) \Rightarrow(i)$, we proceed as follows. Let $\|x\|=\|y\|$. Then $x+y \perp_{\mathbf{i}} x-y$ and therefore $x+y \perp_{\mathrm{J}} x-y$. Thus we have

$$
\|x+y+k(x-y)\| \geq\|x+y\| \text { for all } k \text {. }
$$

In particular for all $\alpha>1$ we have

$$
\left\|x+y+\frac{\alpha^{2}-1}{\alpha^{2}+1}(x-y)\right\| \geq\|x+y\| .
$$

Therefore, for all $\alpha>1$,

$$
\left\|\alpha x+\alpha^{-1} y\right\| \geq \frac{\alpha^{2}+1}{2 \alpha}\|x+y\| \geq\|x+y\|
$$

Thus (iii) implies the following criterion of inner product spaces by Lorch [8]:

$\left(\mathrm{L}_{5}\right) \quad\|x\|=\|y\| \Rightarrow\left\|\alpha x+\alpha^{-1} y\right\| \geq\|x+y\|$ for all $\alpha$. That completes the proof of Theorem 7 .

REMARK 8. A proof of (iii) appears in [3, p. 155] where it is listed as criterion (M). In a paper, Holub [4] has announced the equivalence of (i) and ( $i$ ) without proof in the equivalent form, namely: $x, y \in X$, $\|x\|=\|y\| \Rightarrow x+y \perp_{J} x-y$.

THEOREM 9. Let $X$ be a normed linear space and $0<a, b<1$. Then the following are equivalent:

(i) $X$ is an irmer product space;

(ii) $x, y \in X$ and $\|x+y\|^{2}+\|a x+b y\|^{2}=\|a x+y\|^{2}+\|x+b y\|^{2}$ implies $x \perp_{\mathrm{J}} y$;

(iii) $x, y \in X$ and $x \perp_{J} y$ implies

$$
\|n+,\|^{2}+\|h r+m u\|^{2}=\|h r+u\|^{2}+\|r+m u\|^{2}
$$


For proving this we first prove:

LEMMA 10. Let $X$ be a normed linear space and $0<a, b<1$, $0 \neq x, y \in X$. Then there exists a nomber a such that

$$
\|(\alpha+1) x+y\|^{2}+\|a x+b(\alpha x+y)\|^{2}=\|\alpha x+\alpha x+y\|^{2}+\|x+b(\alpha x+y)\|^{2} .
$$

Proof. Set

$$
\begin{aligned}
g(t)= & \|x+t x+y\|^{2}+\|a x+b t x+b y\|^{2}-\|a x+t x+y\|^{2}-\|x+t b x+b y\|^{2} \\
= & t^{2}\left[\left[\left\|x+\frac{1}{t}(x+y)\right\|^{2}-\|x\|^{2}\right)+\left(\left\|b x+\frac{1}{t}(a x+b y)\right\|^{2}-\|b x\|^{2}\right)\right. \\
& -\left(\left\|x+\frac{1}{t}(a x+y)\right\|^{2}-\|x\|^{2}\right)-\left(\left\|b x+\frac{1}{t}(x+b y)\right\|^{2}-\|b x\|^{2}\right] .
\end{aligned}
$$

Then, for $t \neq 0$,

$$
\begin{aligned}
\frac{g(t)}{t}=(\| x+ & \left.\frac{1}{t}(x+y)\left\|^{2}-\right\| x \|^{2}\right) /(1 / t)+\left(\left\|b x+\frac{1}{t}(a x+b y)\right\|^{2}-\|b x\|^{2}\right) /(1 / t) \\
& -\left(\left\|x+\frac{1}{t}(a x+y)\right\|^{2}-\|x\|^{2}\right) /(1 / t)-\left(\left\|b x+\frac{1}{t}(x+b y)\right\|^{2}-\|b x\|^{2}\right) /(1 / t)
\end{aligned}
$$

and

$$
\begin{aligned}
\lim _{t \rightarrow \pm \infty} \frac{g(t)}{t} & =2\|x\| N_{ \pm}(x, x+y)+2\|b x\| N_{ \pm}(b x, a x+b y) \\
& -2\|x\| N_{ \pm}(x, a x+b y)-2\|b x\| N_{ \pm}(b x, x+b y) \\
& =2\|x\|^{2}(1+a b-a-b)>0 .
\end{aligned}
$$

Here $N_{+}(x, y)$ and $N_{-}(x, y)$ are respectively the right (left) Gâteaux derivatives of the norm at $x$ in the direction of $y$. For the properties of these needed here, see ([6], p. 272). Therefore $g(t) \rightarrow \infty$ as $t \rightarrow+\infty$ and $g(t) \rightarrow-\infty$ as $t \rightarrow-\infty$. Hence there is a number $\alpha$ such that $g(\alpha)=0$, which was to be proved.

Proof of Theorem 9. (i) $\Rightarrow$ (ii) is straight.

(ii) $\Rightarrow$ (iii) We first show that $X$ is strictly convex. If not, choose $x \neq y$ as extreme points of the unit ball of $X$ such that $\|x\|=\|y\|=\left\|\frac{x+y}{2}\right\|=1$. Then

$$
\left\|\frac{x+y}{2}+y\right\|^{2}+\left\|\frac{a(x+y)}{2}+b y\right\|^{2} \neq\left\|a\left(\frac{x+y}{2}\right)+y\right\|^{2}+\left\|\frac{x+y}{2}+b y\right\|^{2} \text {, }
$$


for otherwise $4+(a+b)^{2}=(a+1)^{2}+(b+1)^{2}$, which requires $a=1$ or $\dot{b}=1$. Without loss of generality we assume $a \geq b$. By Lemma 10 choose $\alpha \neq 0$ such that

(15) $\left\|\frac{x+y}{2}+\alpha\left(\frac{x+y}{2}\right)+y\right\|^{2}+\left\|a \frac{x+y}{2}+b\left(\alpha \frac{x+y}{2}+y\right)\right\|^{2}$

$$
=\left\|a \frac{x+y}{2}+\alpha \frac{x+y}{2}+y\right\|^{2}+\left\|\frac{x+y}{2}+b \alpha \frac{x+y}{2}+b y\right\|^{2} \text {. }
$$

Then (ii) implies $\frac{x+y}{2} \perp_{J} \alpha \frac{x+y}{2}+y$; that is

$$
\left\|\frac{x+y}{2}+k \alpha \frac{x+y}{2}+k y\right\| \geq\left\|\frac{x+y}{2}\right\|=1 \text { for all real } k \text {. }
$$

Putting $k=-\frac{1}{\alpha}$ in (16) yields $|\alpha| \leq 1$, and then $k=-\frac{1}{\alpha+2}$ in (16) yields $|\alpha+2| \leq 1$. These two together give $\alpha=-1$. But then (15) gives

$$
1+\left\|\frac{a-b}{2} x+\frac{a+b}{2} y\right\|^{2}=\left\|\frac{a-1}{2} x+\frac{a+1}{2} y\right\|^{2}+\left\|\frac{1-b}{2} x+\frac{1+b}{2} y\right\|^{2} .
$$

Therefore $\left\|\frac{a-1}{2 a} x+\frac{a+1}{2 a} y\right\|=1$. Writing

$$
y=\left(\frac{1-a}{1+a}\right) x+\left(1-\frac{1-a}{1+a}\right)\left(\frac{a-1}{2 a} x+\frac{a+1}{2 a} y\right) \text {, }
$$

We see that $y$ is a convex combination of two points of the unit sphere which is false since $y$ was taken to be an extreme point of the unit ball. Thus $X$ must be strictly convex. Now suppose ( $i i)$ does not imply ( $i i i$ ). Let $x \perp_{J} y$ and

$$
\|x+y\|^{2}+\|b x+a y\|^{2} \neq\|b x+y\|^{2}+\|x+a y\|^{2} .
$$

Choose $\alpha \neq 0$ by Lemma 10 such that

$$
\|\alpha y+x+y\|^{2}+\|a(\alpha y+x)+b y\|^{2}=\|a(\alpha y+x)+y\|^{2}+\|\alpha y+x+b y\|^{2} .
$$

But then $\alpha y+x \perp_{\mathrm{J}} y$, which violates the left uniqueness of Birkhoff-James orthogonality in strictly convex spaces. Hence $(i i) \Rightarrow$ (iii).

(iii) $\Rightarrow$ (i) Let $y \perp_{J} x$. By (iii) and the fact $\alpha y \perp_{J} \beta x$ for all $\alpha$ and $\beta$ we get 


$$
\begin{aligned}
\|y+x\|^{2}= & \|b y+x\|^{2}+\|y+a x\|^{2}-\|b y+a x\|^{2} \\
= & \left(\left\|b^{2} y+x\right\|^{2}+\|b y+a x\|^{2}-\left\|b^{2} y+a x\right\|^{2}\right) \\
& \quad+\left(\|b y+a x\|^{2}+\left\|y+a^{2} x\right\|^{2}-\left\|b y+a^{2} x\right\|^{2}\right)-\|b y+a x\|^{2} \\
= & \left(\left\|b^{2} y+x\right\|^{2}+\left\|y+a^{2} x\right\|^{2}\right)-\left\|b^{2} y+a x\right\|^{2}-\left\|b y+a^{2} x\right\|^{2} \\
\quad & \quad+\left(\left\|b^{2} y+a x\right\|^{2}+\left\|b y+a^{2} x\right\|^{2}-\left\|b^{2} y+a^{2} x\right\|^{2}\right) \\
= & \left\|b^{2} y+x\right\|^{2}+\left\|y+a^{2} x\right\|^{2}-\left\|b^{2} y+a^{2} x\right\|^{2} .
\end{aligned}
$$

By induction one gets that

$$
y \perp_{J} x \Rightarrow\|y+x\|^{2}=\left\|b^{n} y+x\right\|^{2}+\left\|y+a^{n} x\right\|^{2}-\left\|b^{n} y+a^{n} x\right\|^{2}, n \geq 1 .
$$

This in the limit yields

$$
y \perp_{J} x \Rightarrow\|x+y\|^{2}=\|x\|^{2}+\|y\|^{2},
$$

which is sufficient for $X$ to be an inner product space, as seen in Theorem 5.

THEOREM 11. Let $X$ be a normed Zinear space and $0<a, b<1$. Consider the following statements:

(i) $X$ is an inner product space;

(ii) $x, y \in X$ and

$$
\|x+y\|^{2}+\|a x+b y\|^{2}=\|a x+y\|^{2}+\|x+b y\|^{2} \Rightarrow\|x+y\|=\|x-y\| ;
$$

(iii) $x, y \in X$ and

$$
\|x+y\|=\|x-y\| \Rightarrow\|x+y\|^{2}+\|a x+b y\|^{2}=\|a x+y\|^{2}+\|x+b y\|^{2} .
$$

Then $(i) \Rightarrow(i i) \Rightarrow(i i i)$, and $(i i i) \Rightarrow$ (i) when $a=b$.

Proof. (i) $\Rightarrow$ (ii) is straight. To prove $(i i) \Rightarrow(i i i)$ we again first prove that if (ii) holds then $X$ is strictly convex. If not, choose, as before, $x \neq y$ such $x$ and $y$ are extreme points with $\|x\|=\|y\|=\left\|\frac{x+y}{2}\right\|=1$. Assume $a \geq b$. Then as in Theorem 8, use Lemma 10 to get a number $\alpha \neq 0$ such that equation (15) holds. Then, by (ii), we have

$$
\left\|(\alpha+\alpha) \frac{x+y}{2}+y\right\|=\left\|(\alpha-1) \frac{x+y}{2}+y\right\| .
$$


Equation (17) together with the last part of Theorem 1 shows that $|\alpha| \leq 1$. If $0<\alpha \leq 1$, then equation (15) yields

$$
(2+a)^{2}+(a+b(\alpha+1))^{2}=(1+a+a)^{2}+(b a+b+1)^{2},
$$

which is false. If $-1 \leq \alpha<0$, then, from equation (17), we have

$$
(2+\alpha) \leq\left(\frac{1}{2}+\frac{\alpha}{2}\right)+\left(\frac{1}{2}-\frac{\alpha}{2}\right)=1 \text {. }
$$

Thus $\alpha=-1$ is the only possibility. The rest of the argument is the same as in Theorem 9. Thus $X$ is strictly convex. Now suppose ( $i i)$ does not imply (iii). Then there exist points $x$ and $y$ such that $\|x+y\|=\|x-y\| ;$ but

$$
\|x+y\|^{2}+\|a x+b y\|^{2} \neq\|a x+y\|^{2}+\|x+b y\|^{2} .
$$

Then there exists a number $\alpha$ such that

$$
\|x+(\alpha x+y)\|^{2}+\|a x+b(\alpha x+y)\|^{2}=\|a x+(\alpha x+y)\|^{2}+\|x+b(\alpha x+y)\|^{2} .
$$

By (ii) we have

$$
\|x+\alpha x+y\|=\|x-(\alpha x+y)\| .
$$

Thus $x \perp_{i} y$ and $x \perp_{i} \alpha x+y$, which contradicts the uniqueness of isosceles orthogonality proved in Theorem 3.

Now suppose (iii) holds with $a=b$. Let $\|x\|=\|y\|=1$. Then $\|x+y+x-y\|=\|(x+y)-(x-y)\|$ and therefore

$$
\|x+y+x-y\|^{2}+a^{2}\|(x+y)+(x-y)\|^{2}=\|a(x+y)+(x-y)\|^{2}+\|x+y+a(x-y)\|^{2},
$$

or

$$
\left\|\frac{1+a}{2} x-\frac{1-a}{2} y\right\|^{2}+\left\|\frac{1+a}{2} x+\frac{1-a}{2} y\right\|^{2}=1+a^{2}
$$

The result follows from Day's characterization $(D, \sim)$ with $\alpha=\beta=\frac{1+a}{2}$, and $\sim$ replaced by $=$.

REMARK 12. We feel that in Theorem 11, (iii) should imply (i) even when $a \neq b$. It also remains to be seen whether the condition

$$
x, y \in X, x \perp_{p} y \Rightarrow\|a x+b y\|^{2}+\|x+y\|^{2}=\|a x+y\|^{2}+\|x+b y\|^{2}
$$


is sufficient for the norm of $X$ to be an inner product norm.

\section{References}

[1] Mahlon M. Day, "Some characterizations of inner-product spaces", Trans. Amer. Math. Soc. 62 (1947), 320-337.

[2] Mahlon M. Day, "On criteria of Kasahara and Blumenthal for innerproduct spaces", Proc. Amer. Math. Soc. 10 (1959), 92-100.

[3] MahIon M. Day, Normed Zinear spaces, 3rd edition (Ergebnisse der Mathematik und ihrer Grenzgebiete, 21. Springer-Verlag, Berlin, Heidelberg, New York, 1973).

[4] J.R. Holub, "Rotundity, orthogonality, and characterizations of inner product spaces", Bull. Amer. Math. Soc. 81 (1975), 1087-1089.

[5] R.C. James, "Orthogonality in normed linear spaces", Duke Math. J. 12 (1945), 291-302.

[6] Robert C. James, "Orthogonality and linear functionals in normed linear spaces", Trans. Amer. Math. Soc. 61 (1947), 265-292.

[7] P. Jordan and J.V. Neumann, "On inner products in linear, metric spaces", Ann. of Math. (2) 36 (1935), 719-723.

[8] E.R. Lorch, "Certain implication which characterize Hilbert space", Ann. of Math. (2) 49 (1948), 523-532.

[9] K. Sundaresan, "Orthogonality and nonlinear functionals on Banach spaces", Proc. Amer. Math. Soc. 34 (1972), 187-190.

Department of Mathematics,

Indian Institute of Technology,

Kanpur,

Uttar Pradesh,

India. 